\title{
Long-Term Survival in Adult Neuroblastoma with Multiple Recurrences
}

\author{
L. Vénat-Bouvet V. Le Brun-Ly J.Martin O.Gasnier \\ S. Falkowsky N. Tubiana-Mathieu \\ Department of Medical Oncology, CHU Dupuytren, Limoges, France
}

\section{Key Words}

Adults · Long-term survival · Neuroblastoma

\begin{abstract}
Neuroblastoma (NB) rarely occurs in adults, and less than $10 \%$ of the cases occur in patients older than 10 years. Currently, there are no standard treatment guidelines for adult NB patients. We report the case of a young man suffering from NB in adulthood with multiple recurrences. Treatment included multiple resections, chemotherapy, and radiotherapy. This patient remains free of clinical disease more than 7 years after diagnosis.
\end{abstract}

\section{Introduction}

Neuroblastoma (NB) is a tumor derived from the neural crest that usually originates in the adrenal medulla, but it may arise anywhere within the sympathetic nervous system. NB is a common malignancy in children but rarely occurs in adults; less than $10 \%$ of all cases are diagnosed after the age of 10 years. The incidence for patients between 30 and 39 years of age is approximately 0.2 cases per million person-years $[1,2]$. NB has different biological features in adolescents and adults, and a longer course in both of these groups than in children. However, the outcome is poor regardless of stage [3].

We report the effective use of multimodal therapy to achieve long-term survival in adult retroperitoneal NB with multiple recurrences.

\section{Case Report}

In February 2002, a previously healthy 25-year-old man presented with a supraclavicular left node that was completely resected with a diagnosis of neurofibrosarcoma. Physical examination revealed an abdominal mass. Computed tomography (CT) showed a multinodular $13.5 \times 10-\mathrm{cm}$ tumor in the 


\begin{tabular}{c|l|l|l}
$\begin{array}{c}\text { Case Reports in } \\
\text { Oncology }\end{array}$ & $\begin{array}{l}\text { Case Rep Oncol 2010;3:45-48 } \\
\text { D0I: } 10.1159 / 000286142\end{array}$ & & $\begin{array}{l}\text { Published online: February 24, 2010 S. Karger AG, Basel } \\
\text { ISSN 1662-6575 } \\
\text { www.karger.com/cro }\end{array}$ \\
\hline
\end{tabular}

retroperitoneal region, covering the aorta and repulsing the inferior vena cava. No distant metastases were detected. Guided needle biopsy of the lesion revealed adult NB upon histopathological examination at an outside institution. Histologically, the tumor consisted of small and round-shaped pseudorosette-forming cells with hyperchromatic nuclei. Immunohistochemistry showed cytoplasmic positivity for neuronal specific enolase (NSE), chromogranin A, and synaptophysin. A bone scan was negative. Metaiodobenzylguanidine (MIBG) scintigraphy demonstrated an increased uptake by the mass described above. The serum levels of NSE and catecholamine metabolites, including dopamine, were in the normal range. Catecholamine metabolites were not increased in a 24-hour urine collection.

The patient was diagnosed as having stage IV NB. Combination chemotherapy with a CADO/ CDDP-VP16 regimen according to the pediatric protocol was started with cyclophosphamide (CPM $1,500 \mathrm{mg} / \mathrm{m}^{2}$ per cycle), doxorubicin (AD $60 \mathrm{mg} / \mathrm{m}^{2}$ per cycle), vincristine ( $2 \mathrm{mg}$ per cycle), cisplatin (CDDP $100 \mathrm{mg} / \mathrm{m}^{2}$ per cycle), and etoposide (VP16 $500 \mathrm{mg} / \mathrm{m}^{2}$ per cycle) with G-CSF.

After 5 courses of chemotherapy, the mass was surgically removed and a lomboaortic lymphadenectomy performed. The mass was NB with $70 \%$ necrosis. Fluorescence in situ hybridization analysis of the MYCN locus showed no MYCN oncogene amplification. External irradiation of paraaortic lymph nodes was performed postoperatively, delivering 35 Gray (Gy) with 1.8 Gy per day, 5 days per week.

Postoperative MIBG scintigraphy showed no abnormal uptake.

Semestrial follow-up studies, including MIBG scintigraphy, CT, and catecholamine values in the urine and blood were performed.

The first recurrence occurred in 2005, 30 months after the initial surgery. The patient was asymptomatic when he presented for the visit. Physical examination and routine blood levels were normal, but the serum dopamine level was elevated ( $655 \mu \mathrm{g} / \mathrm{g}$ creatinine for normal range 96-463). MIBG scintigraphy demonstrated an increased uptake in the upper mediastinal region, and CT scans of the chest revealed a $25-\mathrm{mm}$ mediastinal mass. The patient underwent thoracotomy and the mass measuring $40 \times 60 \mathrm{~mm}$ was removed completely. Histological and immunohistochemical examination confirmed the diagnosis of NB metastasis.

One month later, the postoperative scintigraphy showed abnormal uptake in the left supraclavicular area, corresponding to a metastatic lymph node. Because MIBG scintigraphy confirmed disease progression, we administrated carboplatin/VP16 (carboplatin $800 \mathrm{mg} / \mathrm{m}^{2}$ per cycle and VP16 500 $\mathrm{mg} / \mathrm{m}^{2}$ per cycle with G-CSF) therapy. After 2 courses of chemotherapy, ${ }^{18} \mathrm{FDG}$ scintigraphy showed a partial response with a diminishing $\mathrm{SUV}_{\max }$ from 5.2 to 3.3. A $3 \mathrm{rd}$ surgical resection was performed, guided by a perioperative detection probe $24 \mathrm{~h}$ after the injection of $5.2 \mathrm{mCi}$ of iodine-123-labeled MIBG.

Postoperative metabolic imaging performed at 1, 3, 6, 12, 15, 21, and 24 months did not reveal any abnormality in connection with the disease. However, the postoperative FDG-TEP in the 15th month showed an increased uptake in the left popliteal area, corresponding to a less than 1-cm nodule localized in the lateral part of the superior extremity of the left leg. Surgical biopsy revealed a hemangioma with schwannoma.

In August 2008, ${ }^{18}$ FDG scintigraphy showed abnormal uptake in the left jugular carotid and nodular pleural uptake on the right, but MIBG scintigraphy was normal. Physical examination was normal and did not find any catecholaminergic manifestations.

In January 2009, FDG-TEP was carried out (33 months after the last surgery) and showed a single lesion, measuring $25 \mathrm{~mm}$ at its greatest diameter, localized in the pleural area with close contact at the 6 th rib and involved the intercostal muscle on the right. An MRI of the chest confirmed the metabolic imaging data, but MIBG scintigraphy was still normal. A thoracotomy was performed and permitted a complete macroscopic resection of the tumor. The histological findings determined that the lesion was a parietal and visceral pleuritic localization of the NB demonstrated with R1 resection. No complications were encountered during the postoperative period. The patient underwent concomitant postoperative radiochemotherapy with cisplatin regimen. The patient is still alive and has no new metastases more than 8 years after diagnosis. 


\begin{tabular}{c|l|l|l}
$\begin{array}{c}\text { Case Reports in } \\
\text { Oncology }\end{array}$ & $\begin{array}{l}\text { Case Rep Oncol 2010;3:45-48 } \\
\text { Dol: } 10.1159 / 000286142\end{array}$ & & $\begin{array}{l}\text { Published online: February 24, 2010 S. Karger AG, Basel } \\
\text { ISSN 1662-6575 } \\
\text { www.karger.com/cro }\end{array}$ \\
\hline
\end{tabular}

\section{Discussion}

Surgical resection, histology, CT, and MIBG scintigraphy are the methods of choice for diagnosis.

In general, catecholamine metabolites in urine or serum, NSE, and lactate dehydrogenase are useful parameters for diagnosis and follow-up. However, an abnormal tumor marker leads to further assessment with detection of relapse or progression in only $14 \%$ of patients [4]. For our patient, the serum dopamine level was elevated only in the first recurrence.

Currently, a reported specificity of $99 \%$ and cumulative sensitivity of roughly $90 \%$ in paraganglioma make ${ }^{123}$ I-MIBG the most important nuclear imaging method [5]. However, false negatives have been reported and may be explained by the absence of catecholamine reuptake and/or dedifferentiated features of malignant tumors [6, 7]. In our case, MIBG scintigraphy was negative in the last recurrence.

For paraganglioma, FDG-PET diagnoses malignancy better than MIBG scintigraphy and reveals more metastases. However, FDG-PET has lower sensitivity and, in particular, lower specificity than MIBG scintigraphy for detecting paragangliomas [8, 9]. Moreover, there are some false positive cases with FDG-TEP, as we reported for our patient.

The perioperative detection probe is a supplementary diagnostic tool for guiding the exeresis of recurrent tumors, which are sometimes difficult to access surgically. Several publications have reported the use of a perioperative detection probe after the injection of MIBG-labeled with iodine-125, or iodine-123, or ${ }^{111}$ In-pentetrotide [10, 11].

Stage III and IV tumors are often initially treated with chemotherapy followed by tumor resection, as was done in our patient [12]. Because of a lack of experience in adults, the treatment followed pediatric protocols. The most frequently used cytostatic agents, either alone or in combination, include alkylating agents (cyclophosphamide/ifosfamide), platinum-based agents (cisplatin/carboplatin), etoposide, adriamycin, and vincaalkaloids.

Clinical data on the survival outcomes of adult patients (defined as 20 years of age and older) with NB are scarce due to the rarity of the disease. Small, single institution reports have described worse outcomes for adults than pediatric patients $[13,14]$. Data from the publicly accessible Surveillance Epidemiology and End Results confirm these findings, but there has been a trend of improved survival for the past decade, which may reflect advancements in treatment [15]. NB in adolescents and adults exhibits different biological characteristics and has a longer course than in children, with multiple recurrences and/or chronic persistent disease [3]. Moreover, MYCN amplification was rarely found.

\section{Conclusion}

Currently, there are no standard treatment guidelines for patients with adult NB. In patients with metastatic disease and at late stages of disease, multimodal therapy should include surgical resection, radiotherapy, and outpatient chemotherapy. Follow-up of patients with NB should include metabolic imaging studies and tumor marker determination. The collection and evaluation of data on adult patients with this rare tumor are warranted in order to optimize treatment strategies. Whether more intensive treatment or myeloablative therapy with stem cell support will improve the outcome of $\mathrm{NB}$ in adults is unclear. 


\section{References}

1 Davis S, Rogers M, Pendergrass T: The incidence and epidemiologic characteristics of neuroblastoma in the United States. Am J Epidemiol 1987;6:1063-1074.

-2 Castleberry RP: Biology and treatment of neuroblastoma. Pediatr Clin North Am 1997;44:919-937.

-3 Francks LM, Bollen A, Seeger RC, Tram DO, Matthay KK: Neuroblastoma in adults and adolescents: an indolent course with poor survival. Cancer 1997;79:2028-2035.

-4 Simon T, Hero B, et al: Tumor markers are poor predictors for relapse or progression in neuroblastoma. Eur J Cancer 2003;39:1899-1903.

5 Brink I, Hoegerle S, Kliisch J, et al: Imaging of pheochromocytoma and paraganglioma. Familial Cancer 2005;4:61-68.

6 Fukuchi K, Inenaga T, Suzuki Y, et al: Paraganglioma seen with FDG Dual-Head Gamma camera coincidence imaging after false-negative results of I-123 MIBG Imaging. Clin Nucl Medicine 2001;26:966-967.

7 Shulkin BL, Koeppe RA, Francis IR, et al: Pheochromocytomas that do not accumulate metaiodobenzylguanidine: localization with PET and administration of FDG. Radiology 1993;186:711.

-8 Pakak K, Eisenhoffer G, Goldstein DS: Functional imaging of endocrine tumors: role of positron emission tomography. Endocrin Reviews 2004;25:568-580.

-9 Ilias I, Yu J, Carrasquillo JA, et al: Superiority of 6- $\left({ }^{18} \mathrm{~F}\right)$-fluorodopamine positron emission tomography versus $\left({ }^{131} \mathrm{I}\right)$-metaiodobenzyyguanidine scintigraphy in the localization of metastatic pheochromocytoma. J Clin Endocrinol Metab 2003;88:4083-4087.

10 Fuertes Manuel J, Mena Gonzales E, Camacho Marti V, et al: 123I-MIBG SPECTCT combined with gamma probe for radioguided localization of pheochromocytoma. Rev Esp Med Nucl 2005;24:418-421.

-11 Ricard M, Tenenbaum F, Schlumberger M, et al: Intraoperative detection of pheochromocytoma with iodine-125 labelled meta-iodobenzylguanidine: a feasibility study. Eur J Nucl Med 1993;20:426-430.

12 Haase GM, Perez C, Atkinson JB: Current aspects of biology, risk assessment, and treatment of neuroblastoma. Semin Surg Oncol 1999;16:91-104.

13 Kushner BH, Kramer K, LaQuaglia MP, et al: Neuroblastoma in adolescents and adults: The Memorian Sloan-Kettering experience. Med Pediatr Oncol 2003;41:508-515.

-14 Tang CK, Hajdu SI: Neuroblastoma in adolescence and adulthood. NY State J Med 1975;75:1434-1438.

15 Esiashvili N, Gooman M, Ward K, Marcus RB Jr, Johnstone PA: Neuroblastoma in adults: incidence and survival analysis based on SEER data. Pediatr Blood Cancer 2007;49:41-46. 